\title{
A kind of non-zero sum mixed differential game of backward stochastic differential equation
}

Huanjun Zhang ${ }^{1 *}$ (D)

"Correspondence:

zhhuanjun@163.com

'School of Control Science and

Engineering, Shandong University,

Jinan, China

\section{Springer}

\begin{abstract}
This paper is concerned with a non-zero sum mixed differential game problem described by a backward stochastic differential equation. Here the term "mixed" means that this game problem contains a deterministic control $v_{1}$ of Player 1 and a random control process $v_{2}$ of Player 2 . By virtue of the classical variational method, a necessary condition and an Arrow's sufficient condition for the mixed stochastic differential game problem are presented. A linear-quadratic mixed differential game problem is discussed, and the corresponding Nash equilibrium point is explicitly expressed by the solution of mean-field forward-backward stochastic differential equation. The most distinguishing feature, compared with the existing literature, is that the optimal state process of the linear-quadratic game satisfies a linear mean-field backward stochastic differential equation. Finally, a home mortgage and wealth management problem is given to illustrate our theoretical results.
\end{abstract}

Keywords: Arrow's sufficient optimality condition; Mean-field backward stochastic differential equation; Non-zero sum mixed differential game; Open-loop equilibrium point

\section{Introduction}

Differential game theory involves multiple individuals (also called players or agents) decision making in the context of dynamical systems. The study of differential game was originally stated by Isaacs [1], and then summed up and developed by Basar and Olsder [2]. The stochastic differential game plays an important role in lots of fields. Many researchers investigated this problem under various setups.

Compared with the development of forward stochastic differential equation (SDE), the study of the backward stochastic differential equation (BSDE) has been going on in the past three decades. The linear case started from Bismut [3] and the basic framework for the nonlinear situation was given by Pardoux and Peng [4]. The theory of BSDE itself has interesting properties, due to these, it is highly desirable to consider the game problem. Hamadène and Lepeltier [5] discussed a stochastic zero sum differential game problem, and investigated the existence of saddle point under Isaacs' condition. Moreover, their results of this problem depend on the solution of BSDE. Based on [5], Wang and Yu [6]

(c) The Author(s) 2020. This article is licensed under a Creative Commons Attribution 4.0 International License, which permits use sharing, adaptation, distribution and reproduction in any medium or format, as long as you give appropriate credit to the original author(s) and the source, provide a link to the Creative Commons licence, and indicate if changes were made. The images or other third party material in this article are included in the article's Creative Commons licence, unless indicated otherwise in a credit line to the material. If material is not included in the article's Creative Commons licence and your intended use is not permitted by statutory regulation or exceeds the permitted use, you will need to obtain permission directly from the copyright holder. To view a copy of this licence, visit http://creativecommons.org/licenses/by/4.0/. 
studied a new non-zero sum stochastic differential game of BSDE, and established a necessary condition and a sufficient condition in the form of a maximum principle for an open-loop equilibrium point. Furthermore, for the same stochastic differential system as in [6], Wang and $\mathrm{Yu}$ [7] dealt with the partial information case. In addition, Shi and Wang [8] studied a non-zero sum stochastic differential game of BSDE with time-delayed generator. Wang et al. [9] discussed asymmetric information linear-quadratic (LQ) non-zero sum differential game of BSDE, and gave the feedback Nash equilibrium points. About other applications of BSDE, please refer to Zhang [10], Li et al. [11], El Karoui et al. [12], and Yong and Zhou [13] for more information. For other developments about mean-field type game, please refer to [14-16]. Different from the above literature, our work has new features as follows:

- The game problem contains two types of control. One is a deterministic control which can impose a deterministic action $v_{1} \in \mathcal{U}_{a d}^{1}$, the other one is a random control process that can impose a random action $v_{2} \in \mathcal{U}_{a d}^{2}$.

- In an LQ problem, the equilibrium point $\left(u_{1}, u_{2}\right)$ is expressed by $\mathbb{E} p$ (see (18) in Sect. 3) and the optimal state satisfies a linear mean-field BSDE.

- In the LQ problem, by introducing mean-field BSDE, which naturally arises from the study of mixed differential games driven by BSDE without mean-field term, we obtain an explicit form of the equilibrium point.

- In the LQ problem, the equilibrium point $\left(u_{1}, u_{2}\right)$ is uniquely obtained by the solution of mean-field forward-backward SDE (19). Due to the above new features, it is difficult to get the existence and uniqueness of (19) in general. We can prove that (19) admits a unique solution under some detailed cases (see Sect. 3).

This paper is inspired by [17], where mixed optimal control of forward SDE rather than BSDE was discussed. Since the construction and property of BSDE are essentially different from those of SDE, the non-zero sum mixed stochastic differential game of BSDE captures different scenarios. See, e.g., Sect. 3 for more information.

The rest of this paper is organized as follows. In Sect. 2, we formulate the model of the non-zero mixed stochastic differential game of BSDE. In Sect. 3, we give the necessary and sufficient conditions for the open-loop equilibrium point of a mixed differential game. In Sect. 4, we use the theoretical results to study an LQ game problem of BSDE, and give an explicit feedback form of equilibrium point. As a practical application, we consider a home mortgage and wealth management problem, and we work out one numerical example with certain particular coefficients in Sect. 5. In Sect. 6, we give some concluding remarks.

\section{Problem formulation and preliminaries}

Throughout this paper, we let $(\Omega, \mathcal{F}, P)$ be a standard probability space with a natural filtration $\left\{\mathcal{F}_{t}, t \geq 0\right\}$ generated by an $\mathcal{F}_{t}$-adapted, $d$-dimensional standard Brownian motion $\left\{\omega_{t}, t \geq 0\right\}$. We denote by $\mathbb{R}^{k}$ the $k$-dimensional Euclidean space, and by $|\cdot|$ and $\langle\cdot, \cdot\rangle$ the norm and the inner product in Euclidean space, respectively. We also denote by $\mathbb{S}^{n}$ the set of symmetric $n \times n$ matrices with real elements, by $C\left([0, T] ; \mathbb{R}^{k}\right)$ the space of $\mathbb{R}^{k}$-valued continuous functions on $[0, T]$, and by $M^{\top}$ and $M^{-1}$ the transpose and the reverse of $M$, 
respectively. For convenience, we introduce several spaces which are used in this paper:

$$
\begin{aligned}
& L^{\infty}\left(0, T ; \mathbb{R}^{k}\right)=\left\{\varphi:[0, T] \rightarrow \mathbb{R}^{k}|\underset{t \in[0, T]}{\operatorname{essup}}| \varphi_{t} \mid<\infty\right\}, \\
& L^{p}\left(0, T ; \mathbb{R}^{k}\right)=\left\{\varphi:\left.[0, T] \rightarrow \mathbb{R}^{k}\left|\int_{0}^{T}\right| \varphi_{t}\right|^{p} d t<\infty\right\}, \quad 1 \leq p<\infty, \\
& L_{\mathcal{F}}^{2}\left(0, T ; \mathbb{R}^{k}\right)=\left\{\varphi:[0, T] \times \Omega \rightarrow \mathbb{R}^{k} \mid \varphi \text { is } \mathcal{F}\right. \text {-progressively measurable and satisfies } \\
&\left.\mathbb{E} \int_{0}^{T}\left|\varphi_{t}\right|^{2} d t<\infty\right\}, \\
& L_{\mathcal{F}_{T}}^{2}\left(\Omega ; \mathbb{R}^{k}\right)=\left\{\xi: \Omega \rightarrow \mathbb{R}^{k} \mid \xi \text { is } \mathcal{F}_{T}\right. \text {-measurable random variable and satisfies } \\
&\left.\mathbb{E}|\xi|^{2}<\infty\right\}, \\
& L_{\mathcal{F}}^{2}\left(\Omega, C\left([0, T] ; \mathbb{R}^{k}\right)\right)=\left\{\varphi:[0, T] \times \Omega \rightarrow \mathbb{R}^{k} \mid \varphi \text { is } \mathcal{F}\right. \text {-adapted, continuous and } \\
&\left.\quad \text { satisfies } \mathbb{E}\left[\sup _{t \in[0, T]}\left|\varphi_{t}\right|^{2}\right]<\infty\right\} .
\end{aligned}
$$

In this paper, we consider a controlled BSDE

$$
\left\{\begin{array}{l}
-d y_{t}^{v_{1}, v_{2}}=g\left(t, y_{t}^{v_{1}, v_{2}}, z_{t}^{v_{1}, v_{2}}, v_{1 t}, v_{2 t}\right) d t-z_{t}^{v_{1}, v_{2}} d w_{t} \\
y_{T}^{\nu_{1}, v_{2}}=\xi
\end{array}\right.
$$

where $g:[0, T] \times \mathbb{R}^{n} \times \mathbb{R}^{n \times d} \times \mathbb{R}^{l_{1}} \times \mathbb{R}^{l_{2}} \rightarrow \mathbb{R}^{n}, \xi \in L_{\mathcal{F}_{T}}^{2}\left(\Omega ; \mathbb{R}^{n}\right), v_{1}$ is a deterministic control of Player 1 , and $v_{2}$ is a random control process of Player 2 . The game system means that these two players work together to achieve a goal $\xi$ at the terminal time $T$.

Let $U_{i}$ be a nonempty convex subset of $\mathbb{R}^{l_{i}}, i=1,2$. We introduce the admissible control set

$$
\begin{aligned}
& \mathcal{U}_{a d}^{1}=\left\{v_{1} \in L^{2}\left(0, T ; \mathbb{R}^{l_{1}}\right) \mid v_{1 t} \in U_{1}, t \in[0, T]\right\}, \\
& \mathcal{U}_{a d}^{2}=\left\{v_{2} \in L_{\mathcal{F}}^{2}\left(0, T ; \mathbb{R}^{l_{2}}\right) \mid v_{2 t} \in U_{2}, t \in[0, T]\right\} .
\end{aligned}
$$

Assumption $1 g\left(\cdot, y, z, v_{1}, v_{2}\right)$ is continuously differentiable in $\left(y, z, v_{1}, v_{2}\right)$. Moreover, the partial derivatives $g_{y}, g_{z}, g_{v_{1}}$ and $g_{v_{2}}$ of $g$ with respect to $y, z, v_{1}$ and $v_{2}$ are uniformly bounded.

If $v_{1}$ and $v_{2}$ are admissible controls and Assumption 1 holds, it follows from [4] that $\operatorname{BSDE}(1)$ admits a unique solution $\left(y^{\nu_{1}, \nu_{2}}, z^{\nu_{1}, \nu_{2}}\right) \in L_{\mathcal{F}}^{2}\left(0, T ; \mathbb{R}^{n}\right) \times L_{\mathcal{F}}^{2}\left(0, T ; \mathbb{R}^{n \times d}\right)$. The nonzero sum mixed stochastic differential game for the two players is that, besides ensuring to achieve the joint pre-given goal $\xi$ at the terminal time $T$, the two players have their own benefits, described by the cost functional

$$
J_{i}\left(v_{1}, v_{2}\right)=\mathbb{E}\left[\int_{0}^{T} l_{i}\left(t, y_{t}^{v_{1}, v_{2}}, z_{t}^{v_{1}, v_{2}}, v_{1 t}, v_{2 t}\right) d t+h_{i}\left(y_{0}^{v_{1}, v_{2}}\right)\right],
$$


where $l_{i}:[0, T] \times \mathbb{R}^{n} \times \mathbb{R}^{n \times d} \times \mathbb{R}^{l_{1}} \times \mathbb{R}^{l_{2}} \rightarrow \mathbb{R}$, and $h_{i}: \mathbb{R}^{n} \rightarrow \mathbb{R}(i=1,2)$ are given continuous functions satisfying the condition

$$
\mathbb{E} \int_{0}^{T}\left|l_{i}\left(t, y_{t}^{\nu_{1}, v_{2}}, z_{t}^{\nu_{1}, \nu_{2}}, v_{1 t}, v_{2 t}\right)\right| d t<+\infty, \quad \text { for all }\left(v_{1}, v_{2}\right) \in \mathcal{U}_{a d}^{1} \times \mathcal{U}_{a d}^{2}
$$

Suppose that each player hopes to minimize his/her cost functional $J_{i}\left(v_{1}, v_{2}\right)$ by selecting an appropriate admissible control $v_{i}(i=1,2)$. Then the problem is to find a pair of admissible controls $\left(u_{1}, u_{2}\right) \in \mathcal{U}_{a d}^{1} \times \mathcal{U}_{a d}^{2}$ such that

$$
\left\{\begin{array}{l}
J_{1}\left(u_{1}, u_{2}\right)=\min _{v_{1} \in \mathcal{U}_{a d}^{1}} J_{1}\left(v_{1}, u_{2}\right), \\
J_{2}\left(u_{1}, u_{2}\right)=\min _{v_{2} \in \mathcal{U}_{a d}^{2}} J_{2}\left(u_{1}, v_{2}\right) .
\end{array}\right.
$$

If there exists $\left(u_{1}, u_{2}\right)$ satisfying (4), we call it an (open-loop) equilibrium point, and denote the corresponding state trajectory by $(y, z)$. We call the above problem a backward non-zero sum mixed stochastic differential game. For simplicity, we denote it by Problem (BNZM).

\section{Necessary and sufficient conditions for the mixed equilibrium point}

Define the Hamiltonian function $H_{1}:[0, T] \times \mathbb{R}^{n} \times \mathbb{R}^{n \times d} \times \mathbb{R}^{l_{1}} \times \mathbb{R}^{l_{2}} \times \mathbb{R}^{n} \rightarrow \mathbb{R}$ by

$$
H_{1}\left(t, y, z, v_{1}, v_{2}, p_{1}\right)=l_{1}\left(t, y, z, v_{1}, v_{2}\right)-\left\langle p_{1}, g\left(t, y, z, v_{1}, v_{2}\right)\right\rangle \text {, }
$$

and $H_{2}:[0, T] \times \mathbb{R}^{n} \times \mathbb{R}^{n \times d} \times \mathbb{R}^{l_{1}} \times \mathbb{R}^{l_{2}} \times \mathbb{R}^{n} \rightarrow \mathbb{R}$ by

$$
H_{2}\left(t, y, z, v_{1}, v_{2}, p_{2}\right)=l_{2}\left(t, y, z, v_{1}, v_{2}\right)-\left\langle p_{2}, g\left(t, y, z, v_{1}, v_{2}\right)\right\rangle \text {, }
$$

where $p_{i}(i=1,2)$ satisfies the following adjoint equation:

$$
\left\{\begin{array}{l}
-d p_{i t}=H_{i y}\left(t, y_{t}, z_{t}, u_{1 t}, u_{2 t}, p_{i t}\right) d t+H_{i z}\left(t, y_{t}, z_{t}, u_{1 t}, u_{2 t}, p_{i t}\right) d \omega_{t}, \\
p_{i 0}=-h_{i y}\left(y_{0}\right)
\end{array}\right.
$$

with $H_{i y}$ and $H_{i z}$ be the partial derivatives of $H$ with respect to $y$ and $z$, respectively.

Now we give the basic assumptions on the cost functional.

Assumption $2 l_{i}\left(\cdot, y, z, v_{1}, v_{2}\right)$ is continuously differentiable in $\left(y, z, v_{1}, v_{2}\right)$, and $h_{i}$ is continuously differentiable with respect to $y(i=1,2)$. Moreover, there exists a constant $C$ such that the partial derivatives $l_{i y}, l_{i z}$ and $l_{i v_{i}}(i=1,2)$ are bounded by $C\left(1+|y|+|z|+\left|v_{1}\right|+\left|v_{2}\right|\right)$.

Assumption 3 For each $\left(v_{1}, v_{2}\right) \in \mathcal{U}_{a d}^{1} \times \mathcal{U}_{a d}^{2}, l_{i}\left(\cdot, y^{\nu_{1}, v_{2}}, z^{v_{1}, v_{2}}, v_{1}, v_{2}\right) \in L_{\mathcal{F}}^{1}(0, T ; \mathbb{R}), l_{i}$ is differentiable in $(y, z)$, and $h_{i}(i=1,2)$ is differentiable with respect to $y$.

Under Assumptions 1-2, it is well known that for (5) there exists a unique solution $p_{i} \in$ $L_{\mathcal{F}}^{2}\left(0, T ; \mathbb{R}^{n}\right)(i=1,2)$, for any given $\left(u_{1}, u_{2}\right)$. 


\subsection{Necessary condition}

Let $\left(v_{1}, v_{2}\right) \in L^{2}\left(0, T ; \mathbb{R}^{l_{1}}\right) \times L_{\mathcal{F}}^{2}\left(0, T ; \mathbb{R}^{l_{2}}\right)$ be given such that $\left(u_{1}+v_{1}, u_{2}+v_{2}\right) \in \mathcal{U}_{a d}^{1} \times \mathcal{U}_{a d}^{2}$. For any $0 \leq \varepsilon \leq 1$, we take the variational controls $u_{1}^{\varepsilon}=u_{1}+\varepsilon v_{1}$ and $u_{2}^{\varepsilon}=u_{2}+\varepsilon v_{2}$.

Since $\mathcal{U}_{a d}^{1}$ and $\mathcal{U}_{a d}^{2}$ are convex, $\left(u_{1}^{\varepsilon}, u_{2}^{\varepsilon}\right) \in \mathcal{U}_{a d}^{1} \times \mathcal{U}_{a d}^{2}$. As illustrated before, we denote by $\left(y^{u_{1}^{\varepsilon}}, z^{u_{1}^{\varepsilon}}\right)$ and $\left(y^{u_{2}^{\varepsilon}}, z^{u_{2}^{\varepsilon}}\right)$ the corresponding state trajectories of game system (1) along with the controls $\left(u_{1}^{\varepsilon}, u_{2}\right)$ and $\left(u_{1}, u_{2}^{\varepsilon}\right)$, respectively. Introduce the variational equation

$$
\left\{\begin{aligned}
&-d \xi_{i t}= {\left[g_{y}\left(t, y_{t}, z_{t}, u_{1 t}, u_{2 t}\right) \xi_{i t}+g_{z}\left(t, y_{t}, z_{t}, u_{1 t}, u_{2 t}\right) \eta_{i t}\right.} \\
&\left.+g_{v_{i}}\left(t, y_{t}, z_{t}, u_{1 t}, u_{2 t}\right) v_{i t}\right] d t-\eta_{i t} d w_{t} \\
& \xi_{i T}=0, \quad i=1,2 .
\end{aligned}\right.
$$

It is easy to see that (6) admits a unique solution $(\xi, \eta) \in L_{\mathcal{F}}^{2}\left(0, T ; \mathbb{R}^{n}\right) \times L_{\mathcal{F}}^{2}\left(0, T ; \mathbb{R}^{n \times d}\right)$ under Assumptions 1-2.

The following lemmas are immediate results of Lemma 2.2 and Lemma 2.3 in Wang and $\mathrm{Yu}$ [7], which play a role in deriving a necessary condition of Problem (BNZM).

Lemma 3.1 If Assumptions 1-2 hold, then we have

$$
\begin{aligned}
& \lim _{\varepsilon \rightarrow 0} \sup _{0 \leq t \leq T} \mathbb{E}\left|\frac{y_{t}^{u^{\varepsilon}}-y_{t}}{\varepsilon}-\xi_{t}\right|^{2}=0, \\
& \lim _{\varepsilon \rightarrow 0} \mathbb{E} \int_{0}^{T}\left|\frac{z_{t}^{u^{\varepsilon}}-z_{t}}{\varepsilon}-\eta_{t}\right|^{2} d t=0 .
\end{aligned}
$$

Since $\left(u_{1}, u_{2}\right)$ is an equilibrium point of problem (BNZM),

$$
\begin{aligned}
& \varepsilon^{-1}\left[J_{1}\left(u_{1}^{\varepsilon}, u_{2}\right)-J_{1}\left(u_{1}, u_{2}\right)\right] \geq 0, \\
& \varepsilon^{-1}\left[J_{2}\left(u_{1}, u_{2}^{\varepsilon}\right)-J_{2}\left(u_{1}, u_{2}\right)\right] \geq 0 .
\end{aligned}
$$

A lemma follows from (7), Lemma 3.1 and Taylor's expansion.

Lemma 3.2 Under Assumptions 1-2, we get

$$
\begin{gathered}
\mathbb{E}\left\{\int _ { 0 } ^ { T } \left[l_{i y}\left(t, y_{t}, z_{t}, u_{1 t}, u_{2 t}\right) \xi_{i t}+l_{i z}\left(t, y_{t}, z_{t}, u_{1 t}, u_{2 t}\right) \eta_{i t}\right.\right. \\
\left.\left.+l_{i v_{i}}\left(t, y_{t}, z_{t}, u_{1 t}, u_{2 t}\right) v_{i t}\right] d t+h_{i y}\left(y_{0}\right) \xi_{i 0}\right\} \geq 0 .
\end{gathered}
$$

Proposition 3.1 Let Assumptions 1-2 hold. Suppose that $\left(u_{1}, u_{2}\right)$ is an equilibrium point of Problem $(B N Z M)$ and $(y, z)$ is the corresponding state trajectory. Then we have

$$
\begin{aligned}
& \mathbb{E}\left|H_{1 v_{1}}\left(t, y_{t}, z_{t}, u_{1 t}, u_{2 t}, p_{1 t}\right), v_{1}-u_{1 t}\right\rangle \geq 0, \\
& \left\langle H_{2 v_{2}}\left(t, y_{t}, z_{t}, u_{1 t}, u_{2 t}, p_{2 t}\right), v_{2}-u_{2 t}\right\rangle \geq 0,
\end{aligned}
$$

for any $\left(v_{1}, v_{2}\right) \in U_{1} \times U_{2}$, where $p_{i}(i=1,2)$ is the solution of $(5)$. 
Proof Applying Itô's formula to $\left\langle\xi_{i}, p_{i}\right\rangle$, we get

$$
\begin{aligned}
\mathbb{E}\left[\xi_{i 0} h_{i y}\left(y_{0}\right)\right] & \\
=- & \mathbb{E} \int_{0}^{T}\left[\left\langle\xi_{i t}, l_{i y}\left(t, y_{t}, z_{t}, u_{1 t}, u_{2 t}\right)\right\rangle+\left\langle\eta_{i t}, l_{i z}\left(t, y_{t}, z_{t}, u_{1 t}, u_{2 t}\right)\right\rangle\right. \\
& \left.+\left\langle p_{i t}, g_{v_{i}}\left(t, y_{t}, z_{t}, u_{1 t}, u_{2 t}\right) v_{i t}\right\rangle\right] d t, \quad i=1,2 .
\end{aligned}
$$

According to Lemma 3.2, (7) and (9), we obtain

$$
\begin{aligned}
& \mathbb{E} \int_{0}^{T}\left\{\left\langle\left[l_{1 v_{1}}\left(t, y_{t}, z_{t}, u_{1 t}, u_{2 t}\right)-p_{1 t} g_{v_{1}}\left(t, y_{t}, z_{t}, u_{1 t}, u_{2 t}\right)\right], v_{1 t}\right\rangle\right. \\
& \left.\quad+\left\langle\left[l_{2 v_{2}}\left(t, y_{t}, z_{t}, u_{1 t}, u_{2 t}\right)-p_{2 t} g_{v_{2}}\left(t, y_{t}, z_{t}, u_{1 t}, u_{2 t}\right)\right], v_{2 t}\right\rangle d t\right\} \geq 0,
\end{aligned}
$$

which implies the desired conclusion. Thus, the proof is complete.

Remark 1 It is worth noting that the necessary condition (8) is different from the cases of $[6,7]$. The difference has interesting application in LQ non-zero sum mixed differential game of BSDE. See, e.g., Theorems 4.1-4.2 below for more details.

\subsection{Sufficient condition}

Proposition 3.2 Let Assumption 1 and Assumption 3 hold. Let $\left(u_{1}, u_{2}\right) \in \mathcal{U}_{a d}^{1} \times \mathcal{U}_{a d}^{2}$ be given such that $l_{i y}\left(\cdot, y, z, u_{1}, u_{2}\right)$ and $l_{i z}\left(\cdot, y, z, u_{1}, u_{2}\right) \in L_{\mathcal{F}}^{2}(0, T)(i=1,2)$. Suppose that the adjoint equation (5) admits a solution $p_{i} \in L_{\mathcal{F}}^{2}\left(0, T ; \mathbb{R}^{n}\right)(i=1,2)$, and

$$
\begin{aligned}
& H_{1}\left(t, y_{t}, z_{t}, u_{1 t}, u_{2 t}, p_{1 t}\right)=\min _{v_{1} \in U_{1}} H_{1}\left(t, y_{t}, z_{t}, v_{1}, u_{2 t}, p_{1 t}\right), \\
& H_{2}\left(t, y_{t}, z_{t}, u_{1 t}, u_{2 t}, p_{2 t}\right)=\min _{v_{2} \in U_{2}} H_{2}\left(t, y_{t}, z_{t}, u_{1 t}, v_{2}, p_{2 t}\right),
\end{aligned}
$$

hold for all $t \in[0, T]$. Moreover, suppose that, for all $(t, y, z) \in[0, T] \times \mathbb{R}^{n} \times \mathbb{R}^{n \times d}$,

$$
\begin{aligned}
& \tilde{H}_{1}(t, y, z)=\min _{v_{1} \in U_{1}} H_{1}\left(t, y, z, v_{1}, u_{2 t}, p_{1 t}\right), \\
& \tilde{H}_{2}(t, y, z)=\min _{v_{2} \in U_{2}} H_{2}\left(t, y, z, u_{1 t}, v_{2}, p_{2 t}\right),
\end{aligned}
$$

exist and are convex in $(y, z)$, and $h_{i}$ is convex in $y(i=1,2)$. Then $\left(u_{1}, u_{2}\right)$ is an equilibrium point of Problem (BNZM).

The proof of Proposition 3.2 is similar to the case that $v_{1}$ and $v_{2}$ are random control processes. We omit the proof here for simplicity. The interested reader is referred to Arrow and Kurz [18] and Wang and Yu [6] for details. This sufficient condition can be called Arrow's sufficient optimality condition for the equilibrium point of Problem (BNZM).

Remark 2 Arrow's sufficient optimality condition provides a valuable tool to certify equilibrium point and generalizes Mangasarian sufficient condition (the sufficiency version of Pontryagin's maximum principle, which is restricted to some control problems). 
In the rest of this section, we use a special case of Problem (BNZM) to show that (10) and (11) are really needed.

Example 3.1 Consider the controlled BSDE $\left(n=d=l_{1}=l_{2}=1\right)$

$$
\left\{\begin{array}{l}
-d y_{t}^{\nu_{1}, v_{2}}=\left(2 v_{1 t}+v_{2 t}\right) d t-z_{t}^{v_{1}, v_{2}} d w_{t}, \\
y_{T}^{\nu_{1}, v_{2}}=\xi
\end{array}\right.
$$

with $U_{1}=U_{2}=[0,+\infty)$ and

$$
\begin{aligned}
& J_{1}\left(v_{1}, v_{2}\right)=\mathbb{E} \int_{0}^{T} \min \left\{v_{1 t}^{2}-3 v_{1 t}, 1\right\} d t+y_{0}^{v_{1}, v_{2}}, \\
& J_{2}\left(v_{1}, v_{2}\right)=\mathbb{E} \int_{0}^{T} \min \left\{v_{2 t}^{2}-3 v_{2 t}, 1\right\} d t+y_{0}^{v_{1}, v_{2}} .
\end{aligned}
$$

The problem is to find the open-loop equilibrium point $\left(u_{1}, u_{2}\right)$. In this example, the Hamiltonian function and the adjoint equation are

$$
\begin{aligned}
& H_{1}\left(t, y, z, v_{1}, v_{2}, p_{1}\right)=\min \left\{v_{1}^{2}-3 v_{1}, 1\right\}-p_{1}\left(2 v_{1}+v_{2}\right), \\
& H_{2}\left(t, y, z, v_{1}, v_{2}, p_{2}\right)=\min \left\{v_{2}^{2}-3 v_{2}, 1\right\}-p_{2}\left(2 v_{1}+v_{2}\right),
\end{aligned}
$$

and

$$
d p_{i t}=0, \quad p_{i 0}=-1, i=1,2
$$

Solving the ordinary differential equation (ODE), we obtain $p_{1 t}=p_{2 t}=-1, t \in[0, T]$. Substituting it into the Hamiltonian function, we get

$$
H_{1}\left(t, y, z, v_{1}, v_{2}, p_{1}\right)= \begin{cases}v_{1}^{2}-v_{1}+v_{2}, & v_{1} \in\left[0, \frac{3+\sqrt{13}}{2}\right), \\ 2 v_{1}+v_{2}+1, & v_{1} \in\left[\frac{3+\sqrt{13}}{2},+\infty\right),\end{cases}
$$

and

$$
H_{2}\left(t, y, z, v_{1}, v_{2}, p_{2}\right)= \begin{cases}v_{2}^{2}-2 v_{2}+2 v_{1}, & v_{2} \in\left[0, \frac{3+\sqrt{13}}{2}\right), \\ 2 v_{1}+v_{2}+1, & v_{2} \in\left[\frac{3+\sqrt{13}}{2},+\infty\right) .\end{cases}
$$

It is easy to check that $H_{i}\left(t, y, z, v_{1}, v_{2}, p_{i}\right)$ is neither a convex nor a concave function of the control $v_{i}$ on the whole time horizon $[0,+\infty), i=1,2$. On the other hand, let $\left(u_{1 t}=\right.$ $\left.\frac{1}{2}, u_{2 t}=1\right), t \in[0, T]$. It is clear that

$$
\begin{aligned}
& \tilde{H}_{1 t}=\min _{v_{1} \in U_{1}} H_{1}\left(t, y, z, v_{1}, u_{2 t}, p_{1 t}\right)=\frac{3}{4}, \\
& \tilde{H}_{2 t}=\min _{v_{2} \in U_{2}} H_{2}\left(t, y, z, u_{1 t}, v_{2}, p_{2 t}\right)=0 .
\end{aligned}
$$

Now all the assumptions required in Proposition 3.2 are satisfied, then $\left(u_{1 t}, u_{2 t}\right)=\left(\frac{1}{2}, 1\right)$, $t \in[0, T]$ is an open-loop equilibrium point. 


\section{An LQ case of Problem (BNZM)}

This section focuses on solving an LQ case of Problem (BNZM). Applying Propositions 3.1-3.2, we obtain an explicit form of the equilibrium point.

Consider a linear BSDE

$$
\left\{\begin{array}{l}
-d y_{t}^{\nu_{1}, v_{2}}=\left[A_{t} y_{t}^{\nu_{1}, v_{2}}+B_{1 t} \nu_{1 t}+B_{2 t} \nu_{2 t}+C_{t} z_{t}^{\nu_{1}, v_{2}}\right] d t-z_{t}^{\nu_{1}, \nu_{2}} d w_{t} \\
y_{T}^{\nu_{1}, v_{2}}=\xi
\end{array}\right.
$$

where $A, B_{1}, B_{2}$ and $C$ are given deterministic matrix-valued functions with proper dimensions.

The class of admissible controls for (12) is

$$
\begin{aligned}
& \mathcal{V}_{a d}^{1}=\left\{v_{1} \in L^{2}\left(0, T ; \mathbb{R}^{l_{1}}\right) \mid v_{1 t} \in \mathbb{R}^{l_{1}}, t \in[0, T]\right\}, \\
& \mathcal{V}_{a d}^{2}=\left\{v_{2} \in L_{\mathcal{F}}^{2}\left(0, T ; \mathbb{R}^{l_{2}}\right) \mid v_{2 t} \in \mathbb{R}^{l_{2}}, t \in[0, T]\right\} .
\end{aligned}
$$

Assumption 4 The coefficients of (12) satisfy

$$
A \in L^{1}\left(0, T ; \mathbb{R}^{n \times n}\right), \quad B_{i} \in L^{2}\left(0, T ; \mathbb{R}^{n \times l_{i}}\right), \quad C \in L^{2}\left(0, T ; \mathbb{R}^{n \times n}\right), \quad i=1,2 .
$$

Let Assumption 4 hold. According to Pardoux and Peng [4], for fixed $\left(v_{1}, v_{2}\right) \in \mathcal{V}_{a d}^{1} \times$ $\mathcal{V}_{a d}^{2}$ and any $\xi \in L_{\mathcal{F}_{T}}^{2}\left(\Omega, \mathbb{R}^{n}\right)$, BSDE (12) has a unique adapted solution $\left(y^{v_{1}, \nu_{2}}, z^{\nu_{1}, v_{2}}\right) \in$ $L_{\mathcal{F}}^{2}\left(0, T ; \mathbb{R}^{n}\right) \times L_{\mathcal{F}}^{2}\left(0, T ; \mathbb{R}^{n \times d}\right)$. Moreover, by a dual technique similar to [12], we have

$$
y_{t}=\mathbb{E}\left[\xi x_{t}^{T}+\int_{t}^{T}\left(B_{1 s} v_{1 s}+B_{2 s} v_{2 s}\right) x_{t}^{s} d s \mid \mathcal{F}_{t}\right]
$$

with

$$
x_{t}^{s}=\exp \left\{\int_{t}^{s}\left(A_{r}-\frac{1}{2} C_{r}^{2}\right) d r+\int_{t}^{s} C_{r} d w_{r}\right\} \geq 0 .
$$

Define the cost functional of the players

$$
\begin{aligned}
J_{i}\left(v_{1}, v_{2}\right)= & \frac{1}{2} \mathbb{E}\left\{\int _ { 0 } ^ { T } \left[\left(y_{t}^{\nu_{1}, v_{2}}\right)^{\top} Q_{i t} y_{t}^{\nu_{1}, v_{2}}+\left(z_{t}^{\nu_{1}, v_{2}}\right)^{\top} K_{i t} z_{t}^{\nu_{1}, v_{2}}\right.\right. \\
& \left.\left.+v_{i t}^{\top} R_{i t} v_{i t}\right] d t+\left(y_{0}^{v_{1}, v_{2}}\right)^{\top} G_{i} y_{0}^{\nu_{1}, v_{2}}\right\}, \quad i=1,2 .
\end{aligned}
$$

Assumption 5 The weighting coefficients in the cost functional (15) satisfy

$$
\begin{cases}Q_{i} \in L^{1}\left(0, T ; \mathbb{S}^{n}\right), & K_{i} \in L^{\infty}\left(0, T ; \mathbb{S}^{n}\right), \\ R_{i} \in L^{\infty}\left(0, T ; \mathbb{S}^{l_{i}}\right), & G_{i} \in \mathbb{S}^{n}\end{cases}
$$

and there exists a constant $\alpha>0$ such that for $t \in[0, T]$

$$
G_{i} \geq 0, \quad R_{i} \geq \alpha I, \quad i=1,2 .
$$


The LQ case of Problem (BNZM) is to find a pair of $\left(u_{1}, u_{2}\right) \in \mathcal{V}_{a d}^{1} \times \mathcal{V}_{a d}^{2}$ such that

$$
\left\{\begin{array}{l}
J_{1}\left(u_{1}, u_{2}\right)=\min _{v_{1} \in \mathcal{V}_{a d}^{1}} J_{1}\left(v_{1}, u_{2}\right) \\
J_{2}\left(u_{1}, u_{2}\right)=\min _{v_{2} \in \mathcal{V}_{a d}^{2}} J_{2}\left(u_{1}, v_{2}\right)
\end{array}\right.
$$

If there exists a pair of $\left(u_{1}, u_{2}\right)$ satisfying (16), then $\left(u_{1}, u_{2}\right)$ is called an equilibrium point of the game. For simplicity, we denote the above problem by problem (MLQ).

According to Proposition 3.1, if $\left(u_{1}, u_{2}\right)$ is an equilibrium point of Problem (MLQ), then the candidate equilibrium point is of the form

$$
\left\{\begin{array}{l}
u_{1 t}=R_{1 t}^{-1} B_{1 t}^{\top} \mathbb{E} p_{1 t} \\
u_{2 t}=R_{2 t}^{-1} B_{2 t}^{\top} p_{2 t}
\end{array}\right.
$$

where the adjoint process $p_{i}$ satisfies

$$
\left\{\begin{array}{l}
d p_{i t}^{\nu_{1}, v_{2}}=\left[A_{t}^{\top} p_{i t}-Q_{i t} y_{t}^{\nu_{1}, \nu_{2}}\right] d t+\left[C_{t}^{\top} p_{i t}-K_{i t} z_{t}^{\nu_{1}, v_{2}}\right] d w_{t}, \\
p_{i 0}^{\nu_{1}, v_{2}}=-G_{i} y_{0}^{\nu_{1}, v_{2}}, \quad i=1,2 .
\end{array}\right.
$$

From Proposition 3.2, we can prove that $\left(u_{1}, u_{2}\right)$ is an open-loop equilibrium point of the game.

We summarize the above deduction in the following theorem.

Theorem 4.1 If $\left(u_{1}, u_{2}\right)$ is an open-loop equilibrium point of Problem $(M L Q)$, then

$$
\left\{\begin{array}{l}
u_{1 t}=R_{1 t}^{-1} B_{1 t}^{\top} \mathbb{E} p_{1 t} \\
u_{2 t}=R_{2 t}^{-1} B_{2 t}^{\top} p_{2 t}
\end{array}\right.
$$

where $p_{i}$ is the solution to the mean-field forward-backward SDE

$$
\left\{\begin{array}{l}
-d y_{t}=\left[A_{t} y_{t}+B_{1 t} R_{1 t}^{-1} B_{1 t}^{\top} \mathbb{E} p_{1 t}+B_{2 t} R_{2 t}^{-1} B_{2 t}^{\top} p_{2 t}+C_{t} z_{t}\right] d t-z_{t} d w_{t} \\
d p_{1 t}=\left[A_{t}^{\top} p_{1 t}-Q_{1 t} y_{t}\right] d t+\left[C_{t}^{\top} p_{1 t}-K_{1 t} z_{t}\right] d w_{t}, \\
d p_{2 t}=\left[A_{t}^{\top} p_{2 t}-Q_{2 t} y_{t}\right] d t+\left[C_{t}^{\top} p_{2 t}-K_{2 t} z_{t}\right] d w_{t} \\
y_{T}=\xi, \quad p_{10}=-G_{1} y_{0}, \quad p_{20}=-G_{2} y_{0} .
\end{array}\right.
$$

Equation (18) is also sufficient for $\left(u_{1}, u_{2}\right)$ to be an open-loop equilibrium point of Problem (MLQ).

Remark 3 The equilibrium point $u_{1}$ in (18) is expressed by $\mathbb{E} p_{1}$ rather than $p_{1}$, this interesting phenomenon is due to the fact that $v_{1}$ is a deterministic control. It is very different from the existing literature; see, e.g., [6].

Note that, since (19) contains the expectation of $p_{1}$, we are uncertain whether (19) admits a unique solution except for some special cases.

In the following, we will use three steps to give the explicit form of Nash equilibrium point of Problem (MLQ). Throughout Sect. 3, we always assume the following. 


\section{Assumption 6}

$$
\left\{\begin{array}{l}
\alpha_{1} B_{1} R_{1}^{-1} B_{1}^{\top}=B_{1} R_{1}^{-1} B_{1}^{\top} \alpha_{1} \\
\alpha_{2} B_{2} R_{2}^{-1} B_{2}^{\top}=B_{2} R_{2}^{-1} B_{2}^{\top} \alpha_{2} \\
B_{1} R_{1}^{-1} B_{1}^{\top}=B_{2} R_{2}^{-1} B_{2}^{\top} \\
C=0
\end{array}\right.
$$

Step 1: Existence and uniqueness of (21).

Under Assumption 6, we have

$$
\left\{\begin{array}{l}
-d y_{t}=\left[A_{t} y_{t}+B_{1 t} R_{1 t}^{-1} B_{1 t}^{\top} \mathbb{E} p_{1 t}+B_{2 t} R_{2 t}^{-1} B_{2 t}^{\top} p_{2 t}\right] d t-z_{t} d w_{t} \\
d p_{1 t}=\left[A_{t}^{\top} p_{1 t}-Q_{1 t} y_{t}\right] d t-K_{1 t} z_{t} d w_{t} \\
d p_{2 t}=\left[A_{t}^{\top} p_{2 t}-Q_{2 t} y_{t}\right] d t-K_{2 t} z_{t} d w_{t} \\
y_{T}=\xi, \quad p_{10}=-G_{1} y_{0}, \quad p_{20}=-G_{2} y_{0} .
\end{array}\right.
$$

Taking the expectation on both sides of (21), we get

$$
\left\{\begin{array}{l}
-d \mathbb{E} y_{t}=\left[A_{t} \mathbb{E} y_{t}+B_{1 t} R_{1 t}^{-1} B_{1 t}^{\top} \mathbb{E} p_{1 t}+B_{2 t} R_{2 t}^{-1} B_{2 t}^{\top} \mathbb{E} p_{2 t}\right] d t \\
d \mathbb{E} p_{1 t}=\left[A_{t}^{\top} \mathbb{E} p_{1 t}-Q_{1 t} \mathbb{E} y_{t}\right] d t \\
d \mathbb{E} p_{2 t}=\left[A_{t}^{\top} \mathbb{E} p_{2 t}-Q_{2 t} \mathbb{E} y_{t}\right] d t, \\
\mathbb{E} y_{T}=\mathbb{E} \xi, \quad \mathbb{E} p_{10}=-G_{1} \mathbb{E} y_{0}, \quad \mathbb{E} p_{20}=-G_{2} \mathbb{E} y_{0} .
\end{array}\right.
$$

According to Assumption 6, we can get the existence and uniqueness of (22). In fact, we introduce an auxiliary equation

$$
\left\{\begin{array}{l}
d Y_{t}=\left[-A_{t} Y_{t}-B_{1 t} R_{1 t}^{-1} B_{1 t}^{\top} P_{t}\right] d t, \\
d P_{t}=\left[A_{t}^{\top} P_{t}-\left(Q_{1 t}+Q_{2 t}\right) Y_{t}\right] d t, \\
Y_{T}=\mathbb{E} \xi, \quad P_{0}=-\left[G_{1}+G_{2}\right] Y_{0},
\end{array}\right.
$$

where $Y=\mathbb{E} y$ and $P=\mathbb{E} p_{1}+\mathbb{E} p_{2}$.

If ( $\left.\mathbb{E} y, \mathbb{E} p_{1}, \mathbb{E} p_{2}\right)$ is a solution to (22), then $(Y, P)$ is a solution to (23). On the other hand, let $(Y, P)$ be a solution to (23). Introduce an ODE

$$
\left\{\begin{array}{l}
d P_{1 t}=\left[A_{t}^{\top} P_{1 t}-Q_{1 t} Y_{t}\right] d t, \\
d P_{2 t}=\left[A_{t}^{\top} P_{2 t}-Q_{2 t} Y_{t}\right] d t, \\
P_{10}=-G_{1} Y_{0}, \quad P_{20}=-G_{2} Y_{0},
\end{array}\right.
$$

which has a unique solution $\left(P_{1}, P_{2}\right)$ with $P_{1}+P_{2}=P$. Furthermore, we can prove that $\left(Y, P_{1}, P_{2}\right)$ is a solution to (22). It implies that the existence and uniqueness of $(22)$ is equivalent to that of (23).

It is easy to check that (23) has a unique solution $(Y, P)$ under Assumptions 4-6 (see Yu and Ji [19]). Then we know that for (22) there exists a unique solution $\left(\mathbb{E} y, \mathbb{E} p_{1}, \mathbb{E} p_{2}\right)$. For 
fixed $\mathbb{E} p_{1}$, from (21), we have

$$
\left\{\begin{array}{l}
-d y_{t}=\left[A_{t} y_{t}+B_{1 t} R_{1 t}^{-1} B_{1 t}^{\top} \mathbb{E} p_{1 t}+B_{2 t} R_{2 t}^{-1} B_{2 t}^{\top} p_{2 t}\right] d t-z_{t} d w_{t} \\
d p_{2 t}=\left[A_{t}^{\top} p_{2 t}-Q_{2 t} y_{t}\right] d t-K_{2 t} z_{t} d w_{t} \\
y_{T}=\xi, \quad p_{20}=-G_{2} y_{0} .
\end{array}\right.
$$

Under Assumptions 4-6, it is clear that fully coupled forward-backward SDE (25) has a unique solution $\left(y, z, p_{2}\right)$ (see, e.g., Theorem 2.3 in [19]). So does (21).

Step 2: The relationship between $y$ and $\left(\mathbb{E} p_{1}, \mathbb{E} p_{2}\right)$.

To get the feedback equilibrium point, we have to establish the relationship between $y$ and $\left(\mathbb{E} p_{1}, \mathbb{E} p_{2}\right)$. Noticing the terminal condition of (22), we set

$$
\mathbb{E} p_{1 t}=\alpha_{1 t} \mathbb{E} y_{t}+\beta_{1 t}, \quad \mathbb{E} p_{2 t}=\alpha_{2 t} \mathbb{E} y_{t}+\beta_{2 t}
$$

Introduce the two ODEs

$$
\left\{\begin{array}{l}
\dot{\alpha_{1 t}}-\alpha_{1 t} A_{t}-A_{t}^{\top} \alpha_{1 t}-\alpha_{1 t} B_{1 t} R_{1 t}^{-1} B_{1 t}^{\top} \alpha_{1 t}-\alpha_{1 t} B_{2 t} R_{2 t}^{-1} B_{2 t}^{\top} \alpha_{2 t}+Q_{1 t}=0 \\
\dot{\beta_{1 t}}-A_{t}^{\top} \beta_{1 t}-\alpha_{1 t} B_{1 t} R_{1 t}^{-1} B_{1 t}^{\top} \beta_{1 t}-\alpha_{1 t} B_{2 t} R_{2 t}^{-1} B_{2 t}^{\top} \beta_{2 t}=0 \\
\alpha_{10}=-G_{1}, \quad \beta_{10}=0
\end{array}\right.
$$

and

$$
\left\{\begin{array}{l}
\dot{\alpha_{2 t}}-\alpha_{2 t} A_{t}-A_{t}^{\top} \alpha_{2 t}-\alpha_{2 t} B_{1 t} R_{1 t}^{-1} B_{1 t}^{\top} \alpha_{1 t}-\alpha_{2 t} B_{2 t} R_{2 t}^{-1} B_{2 t}^{\top} \alpha_{2 t}+Q_{2 t}=0, \\
\dot{\beta_{2 t}}-A_{t}^{\top} \beta_{2 t}-\alpha_{2 t} B_{1 t} R_{1 t}^{-1} B_{1 t}^{\top} \beta_{1 t}-\alpha_{2 t} B_{2 t} R_{2 t}^{-1} B_{2 t}^{\top} \beta_{2 t}=0, \\
\alpha_{20}=-G_{2}, \quad \beta_{20}=0 .
\end{array}\right.
$$

Lemma 4.1 Under Assumption 6, there exists a unique solution $\left(\alpha_{1}, \beta_{1}, \alpha_{2}, \beta_{2}\right)$ to (27) and (28).

Proof Let $\alpha=\alpha_{1}+\alpha_{2}$. It follows from Assumption 6 that

$$
\left\{\begin{array}{l}
\dot{\alpha}_{t}-\alpha_{t} A_{t}-A_{t}^{\top} \alpha_{t}-\alpha_{t} B_{1 t} R_{1 t}^{-1} B_{1 t}^{\top} \alpha_{t}+Q_{1 t}+Q_{2 t}=0, \\
\alpha_{0}=-\left(G_{1}+G_{2}\right) .
\end{array}\right.
$$

Since (29) is a standard Riccati equation, it has a unique solution $\alpha$. Introduce two auxiliary equations

$$
\left\{\begin{array}{l}
\dot{\tilde{\alpha}}_{1 t}-\tilde{\alpha}_{1 t} A_{t}-A_{t}^{\top} \tilde{\alpha}_{1 t}-B_{1 t} R_{1 t}^{-1} B_{1 t}^{\top} \alpha_{t} \tilde{\alpha}_{1 t}+Q_{1 t}=0 \\
\dot{\tilde{\alpha}}_{10}=-G_{1}, \quad t \in(0, T]
\end{array}\right.
$$

and

$$
\left\{\begin{array}{l}
\dot{\tilde{\alpha}}_{2 t}-\tilde{\alpha}_{2 t} A_{t}-A_{t}^{\top} \tilde{\alpha}_{2 t}-B_{2 t} R_{2 t}^{-1} B_{2 t}^{\top} \alpha_{t} \tilde{\alpha}_{2 t}+Q_{2 t}=0, \\
\dot{\tilde{\alpha}}_{20}=-G_{2}, \quad t \in(0, T]
\end{array}\right.
$$


where $\alpha$ is the solution to (29). Obviously, (30) and (31) have unique solutions $\tilde{\alpha}_{1}$ and $\tilde{\alpha}_{2}$, respectively. In addition, we can check that $\alpha_{1}$ and $\alpha_{2}$ in (27) and (28) are also the solutions to (30) and (31), respectively. From the uniqueness of solution of (30) and (31), it follows that

$$
\tilde{\alpha}_{1}=\alpha_{1}, \quad \tilde{\alpha}_{2}=\alpha_{2}
$$

which implies in turn that the first equations of (27) and (28) have the unique solutions $\alpha_{1}$ and $\alpha_{2}$, respectively.

Let $\beta=\beta_{1}+\beta_{2}$ and $\beta_{0}=0$. We have

$$
\left\{\begin{array}{l}
\dot{\beta}_{t}-A_{t}^{\top} \beta_{t}-\alpha_{t} B_{1 t} R_{1 t}^{-1} B_{1 t}^{\top} \beta_{t}=0, \\
\beta_{0}=0
\end{array}\right.
$$

where $\alpha$ is the solution to (29). Note that (32) has a unique solution $\beta$. Introduce

$$
\dot{\tilde{\beta}}_{1 t}-A_{t}^{\top} \tilde{\beta}_{1 t}-\alpha_{1 t} B_{2 t} R_{2 t}^{-1} B_{2 t}^{\top} \beta_{t}=0, \quad \tilde{\beta}_{10}=0, \quad t \in(0, T]
$$

and

$$
\dot{\tilde{\beta}}_{2 t}-A_{t}^{\top} \tilde{\beta}_{2 t}-\alpha_{2 t} B_{1 t} R_{1 t}^{-1} B_{1 t}^{\top} \beta_{t}=0, \quad \tilde{\beta}_{20}=0, \quad t \in(0, T]
$$

where $\alpha_{1}, \alpha_{2}$ and $\beta$ are the solutions to (30), (31) and (32), respectively. Similarly, we can prove that the second equations of (27) and (28) also have unique solutions $\beta_{1}$ and $\beta_{2}$ satisfying

$$
\tilde{\beta}_{1}=\beta_{1}, \quad \tilde{\beta}_{2}=\beta_{2} .
$$

Based on the arguments above, we can derive the unique analytical expressions for $\alpha_{1}, \alpha_{2}$, $\beta_{1}, \beta_{2}$. Then the proof is completed.

\section{Step 3: The relationship between $y$ and $p_{2}$.}

Similarly, we set

$$
p_{2 t}=\Sigma_{t} y_{t}+\Gamma_{t} \mathbb{E} y_{t}+\Phi_{t}
$$

with $\Sigma_{0}=-G_{2}, \Gamma_{0}=0, \Phi_{0}=0$.

Applying Itô's formula to $p_{2}$ in (35), we get

$$
\begin{aligned}
d p_{2 t}= & \left\{\left[\dot{\Sigma}_{t}-\Sigma_{t} A_{t}-\Sigma_{t} B_{2 t} R_{2 t}^{-1} B_{2 t}^{\top} \Sigma_{t}\right] y_{t}\right. \\
& +\left[\dot{\Gamma}_{t}-\Sigma_{t} B_{1 t} R_{1 t}^{-1} B_{1 t}^{\top} \alpha_{1 t}-\Sigma_{t} B_{2 t} R_{2 t}^{-1} B_{2 t}^{\top} \Gamma_{t}\right. \\
& \left.-\Gamma_{t} A_{t}-\Gamma_{t} B_{1 t} R_{1 t}^{-1} B_{1 t}^{\top} \alpha_{1 t}-\Gamma_{t} B_{2 t} R_{2 t}^{-1} B_{2 t}^{\top}\left(\Sigma_{t}+\Gamma_{t}\right)\right] \mathbb{E} y_{t}-\Sigma_{t} B_{1 t} R_{1 t}^{-1} B_{1 t}^{\top} \beta_{1 t} \\
& \left.+\dot{\Phi}_{t}-\Sigma_{t} B_{2 t} R_{2 t}^{-1} B_{2 t}^{\top} \Phi_{t}-\Gamma_{t} B_{1 t} R_{1 t}^{-1} B_{1 t}^{\top} \beta_{1 t}-\Gamma_{t} B_{2 t} R_{2 t}^{-1} B_{2 t}^{\top} \Phi_{t}\right\} d t \\
& +\Sigma_{t} z_{t} d w_{t} .
\end{aligned}
$$


Plugging (35) into (21) yields

$$
d p_{2 t}=\left\{\left[A_{t}^{\top} \Sigma_{t}-Q_{2 t}\right] y_{t}+A_{t}^{\top} \Gamma_{t} \mathbb{E} y_{t}+A_{t}^{\top} \Gamma_{t} \mathbb{E} y_{t}+A_{t}^{\top} \Phi_{t}\right\} d t-K_{2 t} z_{t} d w_{t}
$$

Comparing (36) and (37), we obtain

$$
\begin{aligned}
& \left\{\begin{array}{l}
\dot{\Sigma}_{t}-\Sigma_{t} A_{t}-A_{t}^{\top} \Sigma_{t}-\Sigma_{t} B_{2 t} R_{2 t}^{-1} B_{2 t}^{\top} \Sigma_{t}+Q_{2 t}=0, \\
\Sigma_{0}=-G_{2},
\end{array}\right. \\
& \left\{\begin{array}{l}
\dot{\Gamma}_{t}-\Gamma_{t} A_{t}-A_{t}^{\top} \Gamma_{t}-\left(\Sigma_{t}+\Gamma_{t}\right) B_{1 t} R_{1 t}^{-1} B_{1 t}^{\top} \alpha_{1 t} \\
\quad-\Sigma_{t} B_{2 t} R_{2 t}^{-1} B_{2 t}^{\top} \Gamma_{t}-\Gamma_{t} B_{2 t} R_{2 t}^{-1} B_{2 t}^{\top}\left(\Sigma_{t}+\Gamma_{t}\right)=0, \\
\Gamma_{0}=0,
\end{array}\right.
\end{aligned}
$$

and

$$
\left\{\begin{array}{l}
\dot{\Phi}_{t}-A_{t}^{\top} \Phi_{t}-\Sigma_{t} B_{1 t} R_{1 t}^{-1} B_{1 t}^{\top} \beta_{1 t}-\Sigma_{t} B_{2 t} R_{2 t}^{-1} B_{2 t}^{\top} \Phi_{t} \\
\quad-\Gamma_{t} B_{1 t} R_{1 t}^{-1} B_{1 t}^{\top} \beta_{1 t}-\Gamma_{t} B_{2 t} R_{2 t}^{-1} B_{2 t}^{\top} \Phi_{t}=0, \\
\Phi_{0}=0,
\end{array}\right.
$$

where $\left(\alpha_{1}, \beta_{1}\right)$ is the solution to (27). According to [20], the Riccati equations (38) and (39) admit unique solutions $\Sigma$ and $\Gamma$, respectively. From (26) and (35), we have

$$
\alpha_{2}=\Sigma+\Gamma, \quad \beta_{2}=\Phi .
$$

Since (28) has a unique solution $\left(\alpha_{2}, \beta_{2}\right)$, for (40) there exists a unique solution $\Phi$. Thus, the feedback equilibrium point $\left(u_{1}, u_{2}\right)$ of problem (MLQ) is uniquely defined by

$$
\left\{\begin{array}{l}
u_{1 t}=R_{1 t}^{-1} B_{1 t}^{\top} \alpha_{1 t} \mathbb{E} y_{t}+R_{1 t}^{-1} B_{1 t}^{\top} \beta_{1 t}, \\
u_{2 t}=R_{2 t}^{-1} B_{2 t}^{\top}\left[\Sigma_{t} y_{t}+\Gamma_{t} \mathbb{E} y_{t}+\Phi_{t}\right] .
\end{array}\right.
$$

Substituting (26) and (35) into (21), we obtain

$$
y_{t}=\mathbb{E}\left[\xi x_{T}+\int_{t}^{T} f_{s} x_{s} d s \mid \mathcal{F}_{t}\right] \text {, }
$$

and

$$
\mathbb{E} y_{t}=\mathbb{E} \xi e^{-\int_{t}^{T} g_{r} d r}+\int_{t}^{T} h_{s} e^{\int_{s}^{t} g_{r} d r} d s,
$$

where

$$
\left\{\begin{array}{l}
f_{s}=B_{1 s} R_{1 s}^{-1} B_{1 s}^{\top}\left[\alpha_{1 s}+\Gamma_{s}\right] \mathbb{E} y_{s}+B_{1 s} R_{1 s}^{-1} B_{1 s}^{\top}\left[\beta_{1 s}+\Phi_{s}\right], \\
x_{s}=\exp \left\{\int_{t}^{s}\left[A_{r}+B_{1 r} R_{1 r}^{-1} B_{1 r}^{\top} \Sigma_{r}\right] d r\right\}, \quad s \in[t, T], \\
g_{t}=-A_{t}-B_{1 t} R_{1 t}^{-1} B_{1 t}^{\top}\left[\alpha_{1 t}+\alpha_{2 t}\right], \\
h_{t}=-B_{1 t} R_{1 t}^{-1} B_{1 t}^{\top}\left[\beta_{1 t}+\beta_{2 t}\right] .
\end{array}\right.
$$


We summarize the above deduction in the following theorem.

Theorem 4.2 Let Assumption 6 hold, the feedback equilibrium point $\left(u_{1}, u_{2}\right)$ of problem $(M L Q)$ is uniquely defined by

$$
\left\{\begin{array}{l}
u_{1 t}=R_{1 t}^{-1} B_{1 t}^{\top} \alpha_{1 t} \mathbb{E} y_{t}+R_{1 t}^{-1} B_{1 t}^{\top} \beta_{1 t}, \\
u_{2 t}=R_{2 t}^{-1} B_{2 t}^{\top}\left[\Sigma_{t} y_{t}+\Gamma_{t} \mathbb{E} y_{t}+\Phi_{t}\right],
\end{array}\right.
$$

where $\alpha_{1}, \beta_{1}, \Sigma, \Gamma, \Phi, y$ and $\mathbb{E} y$ are given by (27), (38), (39), (40), (41) and (42), respectively.

Remark 4 We emphasize that the equilibrium point $u_{1}$ depends on $\mathbb{E} y$, and $u_{2}$ depends on $y$ and $\mathbb{E} y$. The main reason of this phenomenon is that $v_{1}$ is a deterministic control and $v_{2}$ is a random control process. This is very different from the case that both $v_{1}$ and $v_{2}$ are random control processes (see, e.g., [6, 7]).

\section{A home mortgage and wealth management problem}

In this section, we study a problem about home mortgage and investment management. This model is inspired by [21], which studied an early assessment of residential mortgage performance in China. Judging from the interest rate difference of various products in China at present, it is reasonable to invest idle funds into some stable wealth management products and return bank loans owed, which can bring certain wealth benefits to buyers and improve the buyers' quality of life. Furthermore, the ability to repay bank loans is affected by factors such as market interest rate, buyers' age, education level, annual income, and building form. We suppose a home buyer intends to repay the bank $v_{1}$ per month, and the home buyer hires a portfolio manager to invest in a risk-free asset and a risky asset. The price processes of the risk-free asset and the risky asset are given by

$$
d I_{1 t}=r_{t} I_{1 t} d t \quad \text { and } \quad d I_{2 t}=\mu_{t} I_{2 t} d t+\sigma_{t} I_{2 t} d w_{t}
$$

respectively.

Now suppose the home buyer plans to attain a terminal wealth goal $\xi$, which is $\mathcal{F}_{T^{-}}$ measure, non-negative, square-integrable random variable. Then the wealth $y_{t}$ is modeled by

$$
\left\{\begin{array}{l}
d y_{t}=\left\{r_{t} y_{t}+a_{t} z_{t}-v_{1 t}-v_{2 t}\right\} d t+z_{t} d w_{t} \\
y_{T}=\xi
\end{array}\right.
$$

where $z_{t}=\pi_{t} \sigma_{t}, a_{t}=\left(\mu_{t}-r_{t}\right) \sigma_{t}^{-1}, \pi_{t}$ is the amount that the portfolio manager invests in the risk asset, $v_{1}$ is the strategy of repaying home mortgage, $v_{2}$ is the instantaneous consumption rate of the portfolio manager. From another perspective, the home buyer is another portfolio manager. We assume that the market coefficients $r_{t}, \mu_{t}, \sigma_{t}$ are deterministic and bounded processes, and $\sigma_{t}^{-1}$ is also bounded. Note that the strategy of repaying home mortgage is deterministic over a certain period of time. Here, we set

$$
\begin{aligned}
& \mathcal{H}_{a d}^{1}=\left\{v_{1} \in L^{2}(0, T ; \mathbb{R}) \mid v_{1 t}>0, t \in[0, T]\right\}, \\
& \mathcal{H}_{a d}^{2}=\left\{v_{2} \in L_{\mathcal{F}}^{2}(0, T ; \mathbb{R}) \mid v_{2 t}>0, t \in[0, T]\right\},
\end{aligned}
$$


every element of $\mathcal{H}_{i}$ is called an admissible control. The coefficients of (44) satisfy Assumption 4 , then we get

$$
y_{t}=\mathbb{E}\left[\xi \zeta_{T}+\int_{t}^{T}\left(v_{1 s}+v_{2 s}\right) \zeta_{s} d s \mid \mathcal{F}_{t}\right]
$$

where

$$
\left\{\begin{array}{l}
d \zeta_{s}=-r_{s} \zeta_{s} d s-a_{s} \zeta_{s} d w_{s}, \quad t \leq s \leq T \\
\zeta_{t}=1
\end{array}\right.
$$

From (47), we get

$$
\zeta_{s}=\exp \left\{\int_{t}^{s}\left[-r_{\tau}-\frac{1}{2} a_{\tau}^{2}\right] d \tau-\int_{t}^{s} a_{\tau} d w_{\tau}\right\} \geq 0
$$

Furthermore, if the terminal condition $\xi$ is non-negative, then from (46) and (48), we get the solution of (44) is larger than zero. We define the associated cost functional of the portfolio managers

$$
J_{i}\left(v_{1}, v_{2}\right)=-\mathbb{E} \int_{0}^{T} C_{i} e^{-\beta t} \ln v_{i t} d t+\theta_{i} y_{0}, \quad i=1,2,
$$

where $\beta$ is a discount factor, $C_{i}$ and $\theta_{i}(i=1,2)$ are positive constants. In (49), the first term measures the total utility from $v_{i}(i=1,2)$, and the last term represents the initial reserve. That is to say, the home buyer desires to maximize the expected utility as well as to minimize the initial reserve. Then our problem is to find an equilibrium point $\left(u_{1}, u_{2}\right) \in$ $\mathcal{H}_{a d}^{1} \times \mathcal{H}_{a d}^{2}$ such that

$$
\left\{\begin{array}{l}
J_{1}\left(u_{1}, u_{2}\right)=\min _{v_{1} \in \mathcal{H}_{a d}^{1}} J_{1}\left(v_{1}, u_{2}\right), \\
J_{2}\left(u_{1}, u_{2}\right)=\min _{v_{2} \in \mathcal{H}_{a d}^{2}} J_{2}\left(u_{1}, v_{2}\right) .
\end{array}\right.
$$

We use Proposition 3.1 to guess a candidate equilibrium point

$$
\left\{\begin{array}{l}
u_{1 t}=C_{1} e^{-\beta t}\left(\mathbb{E} p_{1 t}\right)^{-1}, \\
u_{2 t}=C_{2} e^{-\beta t}\left(p_{2 t}\right)^{-1}
\end{array}\right.
$$

and the adjoint process $p_{i t}$ satisfies

$$
\left\{\begin{array}{l}
d p_{i t}=-r_{t} p_{i t} d t-a_{t} p_{i t} d w_{t}, \\
p_{i 0}=\theta_{i}, \quad i=1,2 .
\end{array}\right.
$$

Solving (52), we get

$$
\mathbb{E} p_{1 t}=\theta_{1} \exp \left\{\int_{0}^{t}-r_{s} d s\right\}>0,
$$




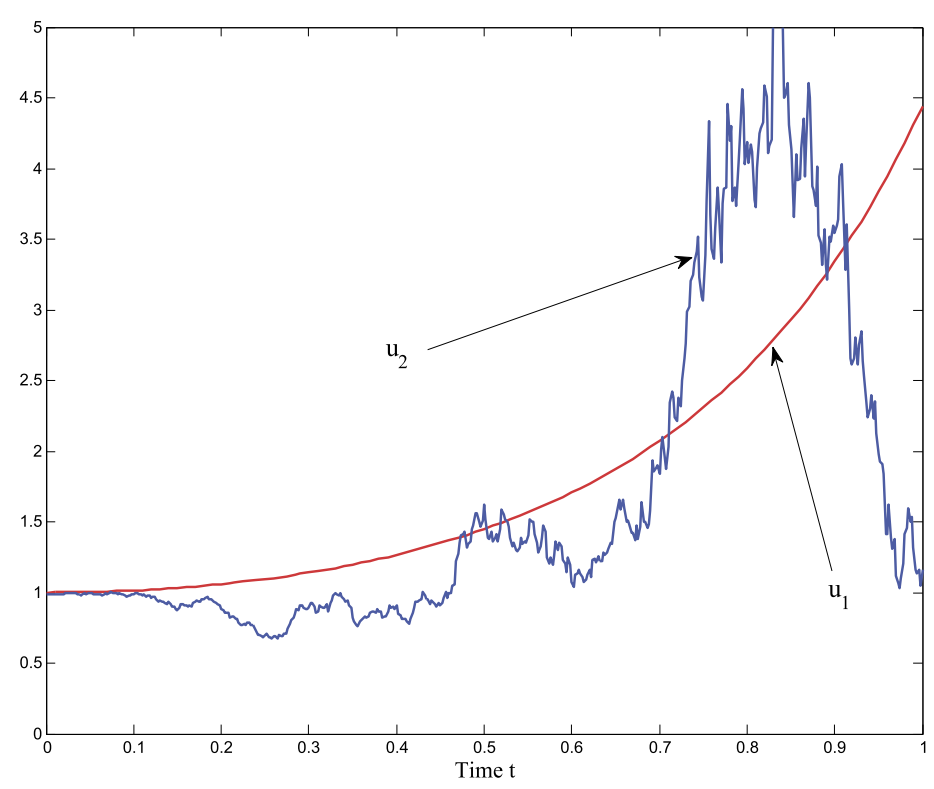

Figure 1 One trajectory of $u_{1}$ and $u_{2}$

$$
p_{2 t}=\theta_{2} \exp \left\{\int_{0}^{t}\left[-r_{s}-\frac{1}{2} a_{s}^{2}\right] d s-\int_{0}^{t} a_{s} d w_{s}\right\}>0 .
$$

From the assumption of $C_{1}, \beta, \theta_{1}$, and $r_{t}$, we see that $u_{1}$ is square integrable. Applying a standard exponential martingale property to $u_{2}$, we can check that $u_{2}$ is square integrable. Then we get $\left(u_{1}, u_{2}\right) \in \mathcal{H}_{a d}^{1} \times \mathcal{H}_{a d}^{2}$. Assumption 3 is weaker than Assumption 2, and all the conditions in Proposition 3.2 are satisfied, then Proposition 3.2 implies that $\left(u_{1}, u_{2}\right)$ is an equilibrium point of the home mortgage and wealth management problem. Putting $\left(u_{1}, u_{2}\right)$ into (46) and (49), we get the corresponding initial wealth and related utilities, respectively.

We illustrate the above theoretical results by working out one numerical example with certain particular coefficients. Let $r_{t}=3 t, a_{t}=2 t, \beta=0.01, C_{1}=1, C_{2}=2, \theta_{1}=1, \theta_{2}=2$.

Applying the Runge-Kutta method and the Monte Carlo method, we obtain the dynamic simulation of $\left(\cdot, w, u_{1}, u_{2}\right)$. For simplicity, we only draw the trajectory of $\left(u_{1}, u_{2}\right)$, shown in Fig. 1.

\section{Conclusion}

Motivated by the lack of theory and some interesting financial and economic phenomena, in this paper, we study a non-zero sum mixed differential game of BSDE. We establish a necessary condition and an Arrow sufficient condition for open-loop equilibrium point. There are two contributions worthy of being highlighted. One is that the equilibrium point $\left(u_{1}, u_{2}\right)$ can be explicitly expressed under some detailed conditions. The other one is that the mixed feedback equilibrium point $\left(u_{1}, u_{2}\right)$ not only depends on the optimal state but also depends on its expectation (see (43)). Due to these features, this paper differs from the existing literature.

Here, we only study the non-zero sum mixed differential game of BSDE under complete information. Extension of our problem formulation to other type of mixed stochas- 
tic differential game problem promises to be interesting research topics, e.g., the mixed stochastic differential game of BSDE with partial information, and the mixed stochastic differential game of backward stochastic differential delay equation. We will consider these topics in our future research.

\section{Acknowledgements}

The author would like to thank Professor Guangchen Wang for his constructive and insightful comments for improving the quality of this work.

\section{Funding}

This work was supported in part by the National Natural Science Foundation of China under Grants 11371228,61821004 61633015, by the National Natural Science Foundation for Excellent Young Scholars of China under Grant 61422305, by the National Natural Science Fund for Distinguished Young Scholars of China under Grant 61925306, and by the Young Chang Jiang Scholars Program of Chinese Education Ministry.

\section{Abbreviations}

$B S D E$, backward stochastic differential equation; LQ, linear-quadratic; SDE, stochastic differential equation; ODE, ordinary differential equation.

\section{Availability of data and materials}

Not applicable.

\section{Competing interests}

The author declares that there is no conflict of interests regarding the publication of this paper

\section{Authors' contributions}

The author read and approved the final manuscript.

\section{Publisher's Note}

Springer Nature remains neutral with regard to jurisdictional claims in published maps and institutional affiliations.

Received: 9 May 2019 Accepted: 9 January 2020 Published online: 20 January 2020

\section{References}

1. Isaacs, R.: Differential games, Parts 1-4. The Rand Corporation, Research Memorandums Nos. RM-1391, RM-1411, RM-1486 55 (1954)

2. Basar, T., Olsder, G.J.: Dynamic Noncooperative Game Theory. Academic Press, London (1982)

3. Bismut, J.M.: An introductory approach to duality in optimal stochastic control. SIAM Rev. 20(1), 62-78 (1978)

4. Pardoux, E., Peng, S.: Adapted solution of backward stochastic differential equation. Syst. Control Lett. 14(1), 55-61 (1990)

5. Hamadène, S., Lepeltier, J.P.: Zero-sum stochastic differential games and backward equations. Syst. Control Lett. 24(4), 259-263 (1995)

6. Wang, G., Yu, Z.: A Pontryagin's maximum principle for non-zero sum differential games of BSDEs with applications. IEEE Trans. Autom. Control 55(7), 1742-1747 (2010)

7. Wang, G., Yu, Z.: A partial information non-zero sum differential game of backward stochastic differential equations with applications. Automatica 48(2), 342-352 (2012)

8. Shi, J., Wang, G.: A non-zero sum differential game of BSDE with time-delayed generator and applications. IEEE Trans. Autom. Control 61(7), 1959-1964 (2016)

9. Wang, G., Xiao, H., Xiong, J.: A kind of linear quadratic non-zero sum differential game of backward stochastic differential euqation with asymmetric information. Automatica 97, 346-352 (2018)

10. Zhang, F.: Maximum principle for near-optimality of stochastic delay control problem. Adv. Differ. Equ. 2017, 98 (2017). https://doi.org/10.1186/s13662-017-1155-9

11. Li, X., Sun, J., Xiong, J.: Linear quadratic optimal control problems for mean-field backward stochastic differential equations. Appl. Math. Optim. 80, 223-250 (2019)

12. El Karoui, N., Peng, S., Quenez, M.C.: Backward stochastic differential equations in finance. Math. Finance 7(1), 1-71 (1997)

13. Yong, J., Zhou, X.: Stochastic Controls: Hamiltonian Systems and HJB Equations. Springer, New York (1999)

14. Djehiche, B., Hamadène, S.: Optimal control and zero-sum stochastic differential game problems of mean-field type. Appl. Math. Optim. (2018). https://doi.org/10.1007/s00245-018-9525-6

15. Djehiche, B.. Hamadène, S.: Mean-field backward-forward stochastic differential equations and non zero sum stochastic diferential games (2019) arXiv:1904.06193

16. Duncan, T., Hamidou, T.: Linear quadratic mean field type games: a direct method. Games 9, 7 (2018). https://doi.org/10.3390/g9010007

17. Hu, Y., Tang, S.: Mixed deterministic and random optimal control of linear stochastic systems with quadratic costs. Probab. Uncertain. Quant. Risk 4, 1 (2019). https://doi.org/10.1186/s41546-018-0035-X

18. Arrow, K.J., Kurz, M.: Public Investment, the Rate of Return, Optimal Fiscal Policy. John Hopkins Press, Baltimore (1970)

19. Yu, Z., Ji, S.: Linear-quadratic nonzero-sum differential game of backward stochastic differential equations. In: Proceedings of the 27th Chinese Control Conference, Kunming, pp. 562-566 (2008) 
20. Lim, A.E.B., Zhou, X.: Linear-quadratic control of backward stochastic differential equations. SIAM J. Control Optim. 40(2), 450-474 (2001)

21. Deng, Y., Zheng, D., Ling, C.: An early assessment of residential mortgage performance in China. J. Real Estate Finance Econ. 31(2), 117-136 (2001)

Submit your manuscript to a SpringerOpen ${ }^{\circ}$ journal and benefit from:

- Convenient online submission

- Rigorous peer review

- Open access: articles freely available online

- High visibility within the field

- Retaining the copyright to your article

Submit your next manuscript at $\gg$ springeropen.com 